\title{
MAPK/ERK Dysfunction in Neurodegenerative Diseases
}

Created by: Hector Albert Gasco ${ }^{1}$, Esther Castillo-gÓmez ${ }^{2}$, Francisco Ros-bernal ${ }^{3}$, (Prancisco E Olucha-bordonau 4

1, UK Dementia Research Institute at the University of Cambridge and Department of Clinical Neurosciences, University of Cambridge, Cambridge; ha437@medschl.cam.ac.uk

2, Department of Medicine, School of Medical Sciences, Universitat Jaume I, Castellón de la

Plana, Spain Spanish National Network for Research in Mental Health, Centro de Investigación

Biomédica en Red de Salud Mental (CIBERSAM), Madrid, Spain; escastil@uji.es

3, UP Medicina, Fac CC Salut, Universitat Jaume I, Castelló de la Plana; fros@uji.es

4, UP Medicina, Fac CC Salut, Universitat Jaume I, Castelló de la Plana; folucha@uji.es

Version received: 30 June 2020

check for updates

The signaling pathway of the microtubule-associated protein kinase or extracellular regulated kinase (MAPK/ERK) is a common mechanism of extracellular information transduction from extracellular stimuli to the intracellular space. The transduction of information leads to changes in the ongoing metabolic pathways and the modification of gene expression patterns. In the central nervous system, ERK is expressed ubiquitously, both temporally and spatially. The MAP-ERK pathway is a key element of the neuroinflammatory pathway triggered by glial cells during the development of neurodegenerative diseases, such as Parkinson's and Alzheimer's disease, Huntington's disease, and amyotrophic lateral sclerosis, as well as prionic diseases. The process triggered by MAPK/ERK activation depends on the stage of development (mature or senescence), the type of cellular element in which the pathway is activated, and the anatomic neural structure. However, extensive gaps exist with regards to the targets of the phosphorylated ERK in many of these processes.

MAPK signaling pathways intervene and control cellular functions, resulting in a direct function of memory and emotional processes. Therefore, alterations or modulations of these pathways can lead to different processes implicated in various human diseases. Throughout this and the next section, we will analyze the state-of-the-art of MAPK signaling pathways in human disease, with a special focus on neurodegenerative disorders (Table 1).

The role of the MAPK/ERK pathway in neurodegenerative diseases is mainly related to glial cell function and the inflammatory response. The activation of resident immune cells of the brain, glial cells (microglia and astroglia), triggers the pro-inflammatory state with the production of nitric oxide (NO), cytokines, and chemokines and the implication of inflammatory-related pathways $[1[2][3]$. Most of the components of these pathways are cytosolic targets of ERK, suggesting an essential function of the MAPK pathway in the production or sustaining of such a pathological hallmark, and consequently, in the noxious events that lead to the specific neurodegeneration.

\section{Parkinson's Disease}

Parkinson's disease (PD) is an age-associated disease mostly identified by an extrapyramidal alteration of movement. From a pathological point of view, PD is characterized by the selective and progressive loss of dopaminergicmelanized neurons located in caudoventral regions of the substantia nigra, reactive gliosis, and intracytoplasmic inclusions of a-synuclein known as the Lewy bodies [4]. In this sense, $\alpha$-Synuclein promotes inflammation via activating p38, ERK, and JNK pathways in human microglial cells, resulting in the production of IL-1 $1 \beta$ and TNF- $\alpha$. The disappearance of neurons in the substantia nigra leads to dopamine deficiency in their target areas (in the striatum and other nuclei of the basal ganglia), producing serial functional lesions and the manifestation of symptoms and clinical signs. Many genes, including 23 genes or loci linked to rare monogenic familial forms of PD with Mendelian inheritance, such as SNCA, Parkin, DJ-1, PINK 1, LRRK2, and VPS35, and over 20 common variants with small effect sizes and 12 genetic risk factors, have been associated with PD in recent years [5]6].

Table 1. Participation of Erk in neurodegenerative diseases. 
Parkinson's disease

References

[7][8][9]

LRRK2

6-OHDA model

6-OHDA elicits sustained ERK phosphorylation related to LID

[10][11]

MPTP model

PD patients
[15][16][17]

[18]

[19]

[20][21][22][23]
AD patients

$A D$ patients

SH-SY5Y cells

PC12 cells

Transgenic mice

ERK-signaling induces $A \beta$-associated behavioral deficits

\section{Alzheimer's disease}

Ab dysregulates hippocampal ERK

$\alpha 7 n A C h$ induce tau phosphorylation and neurofibrillary tangle formation after binding to soluble $\mathrm{Ab}$

$\mathrm{HO} 1$ protects against $A \beta$-induced oxidative stress

\section{ALS and HD}

SOD1 transgenic mice

Mutant Htt model

$$
\begin{aligned}
& \text { ERK deficien } \\
& \text { susceptibility }
\end{aligned}
$$

ERK is down regulated, which induces a dysregulation in axonal transport

[24]

\section{Prion diseases}

Prion infected mice
ERK is neuroprotective following prion infection 
idiopathic PD [8]. In this sense, the G2019S mutation is the most common pathogenic mutation associated with the familial form of PD, representing about $3 \%$ of cases overall (40\% in some populations). The LRRK2 locus has also been associated with idiopathic PD (iPD), as an oxidative mechanism selectively increased wild-type LRRK2 kinase in both the substantia nigra from iPD patients and in two different rat models of the disease [29]. Although all MAPKs participate in neurodegeneration associated with LRRK2, ERK is the most plausible downstream mediator of mutant LRRK2 effects [7]. In this regard, it has been observed that the dysregulation of dopaminergic neurodegenerationrelated genes in induced pluripotent stem cells derived from PD patients harboring a G2019S mutation could be minimized by ERK inhibitors [8]. Additionally, during the last decade, an increase in pERK in leucocytes from patients carrying the G2019S mutation [9], the presence of cytoplasmic granules of pERK in Lewy body aggregates in the substantia nigra of LRRK2 G2019S PD patients [7], and a G2019S-LRRK2-associated neurite retraction triggered by ERK-dependent mechanisms in a PD in vitro model have been described $[30]$.

The impact of ERK in PD-associated neurodegeneration has also been analyzed using the most relevant animal models of parkinsonism, both neurotoxins 6- OHDA and 1-Methyl-4-phenyl-1, 2,3,6-tetrahydropyridine (MPTP), suggesting that ERK may contribute to the pathogenesis of neurodegeneration.

6-OHDA remains the most widely used tool to induce a selective nigrostriatal lesion in murine models and dopaminergic cell lines. In this regard, B65 6-OHDA-induced cell death depends on chronic ERK activation, and this dopaminergic death can be mostly attenuated using an MEK blocker [10]. Conversely, when the same cell line is treated which hydrogen peroxide, also inducing transient ERK activation, MEK blockers are ineffective for modifying cell death ${ }^{[10]}$. Moreover, a recent study revealed the inhibition of L-DOPA-induced dyskinesias (LID) following the counteraction of ERK in the dopamine-depleted striatum of 6-OHDA-treated mice [11].

MPTP is a compound that, having passed through the blood-brain barrier, is catabolized by astrocytes to its neurotoxic form MPP+ and causes permanent symptoms of parkinsonism selectively affecting dopaminergic neurons in the substantia nigra. The addition of MPP + to neuroblastoma cell lines increases a-synuclein, induces the activation of ERK, and triggers cell death that can be reverted using the MEK-P inhibitor U0126 [12]. As the action of this inhibitor excludes altering a-synuclein levels, it seems that both ERK activation and a-synuclein pathways are independent [31]. In this sense, ERK is almost exclusively activated in the microglia localized in striatum and substantia nigra pars compacta of MPTP-treated mice [32]. Moreover, the administration of Galectin-1, with an anti-neuroinflammatory effect, to MPTP-treated mice resulted in microglial p38 and ERK1/2 dephosphorylation, followed by IKB/NFKB signaling pathway inhibition ameliorating the neurodegenerative process [13].

Finally, the implication of the ERK pathway in PD is beyond animal models. The substantia nigra of PD patients presents phosphorylated-ERK associated with fibrillar bundles inside coarse discrete cytoplasmic granular accumulations surrounding Lewy bodies, suggesting a potential interaction between the mitochondrial function and the MAPK/ERK signaling pathway in dopaminergic neurodegeneration [14].

\section{Alzheimer's Disease}

Alzheimer's disease (AD) is the most common form of dementia and the most prevalent neurodegenerative disease [33]. AD is a neurodegenerative disorder of an unknown etiology characterized by the progressive loss of memory and other cognitive functions that lead to dementia. The brains of AD patients have several distinctive neuropathological features: Intracellular neurofibrillary tangles (NFTs), whose main component is the abnormally phosphorylated tau protein [34]; senile plaques $(S P)$, primarily consisting of beta-amyloid $(A \beta)^{[35]}$; and neurodegeneration [36], especially relevant in the basal telencephalon, the origin of cortical and hippocampal cholinergic innervation [37][38]. Besides, the disease progresses through a reduction of synaptic proteins [39], changes in the synaptic morphology and structure ${ }^{[35]}$, and neuroinflammation $[40]$. AD usually occurs sporadically, but approximately $5-10 \%$ of patients manifest it in a familiar way.

MAPK pathways differentially activate during AD. All three MAP-kinases are implicated in mild and severe cases (Braak stages III-VI), both ERK and JNK/SAPK are implicated in Braak stages I and II and in non-demented cases without pathology hallmarks (Braak stage 0), and either ERK alone or JNK/SAPK alone can be activated [41]. This different participation suggests that both oxidative stress (JNK/SAPK and p38) and mitotic signaling alterations (ERK) 
are independently able to initiate, but both are necessary to disseminate, disease pathogenesis.

Amyloid b, the principal component of amyloid plaques, constitutes the main link with ERK pathway activation. In this sense, it has been established in both in vivo and in vitro studies that chronically elevated levels of Ab induce the dysregulation of hippocampal ERK MAPK [15][16]. Additionally, increased p-ERK was revealed in brain extracts of AD patients [17]. On the other hand, the oxidative stress induced by Ab activates p38 MAPK and triggers the hyperphosphorylation of tau, which is the other main neuropathological hallmark in AD [42].

Interacting with both $\mathrm{AD}$-associated proteins, the $\alpha 7$ nicotinic acetylcholine receptor ( $\alpha 7 \mathrm{nAChR})$ binds to soluble amyloid-beta, resulting in tau phosphorylation and the formation of neurofibrillary tangles. Moreover, $\alpha 7 n A C h R$ mediates the activation of p38 MAPK and ERK1/2 signaling pathways, suggesting an essential role of both a7nAChR and MAPK signaling pathways in the uptake and accumulation of b-amyloid [18].

Furthermore, during the last decade, it has been suggested that mitochondrial dysfunction is an early pathological feature of $A D$ related to oxidative stress and $\mathrm{Ca} 2+$ homeostasis that triggers $A b$-induced synaptic dysfunction [43]. It has been proved that heme oxygenase-1 (HO-1) plays a role in protecting neurons against $A \beta$-induced oxidative stress [19]. Recent studies have demonstrated that acteoside induces HO-1 expression through Nrf2 activation. This activation depends on ERK and PI3K/Akt pathways, but not on JNK and p38MAPK pathways [44].

However, the role of ERK in AD is not clear, since an increase of total ERK, specifically within synaptosomes, is associated with a deficient memory task performance in AD transgenic mice [20]. In this sense, the activation of ERK, downstream of NMDA NR2B receptor activity, plays an interesting role in regulating memory processes [21]. Moreover, alterations in NR2B phosphorylation and MAPK/ERK signaling induce beta amyloid-associated behavioral deficits in an AD murine model [22]. Recently, it has been demonstrated that changes in synaptosome MAPK/ERK signaling following ACE2-activator administration increased signaling through the NR2B receptor, inducing significant protection against cognitive decline and decreasing the amyloid accumulation [23].

\section{Amyotrophic Lateral Sclerosis and Huntington's Disease}

Amyotrophic lateral sclerosis (ALS), a term proposed by Charcot in 1874[45], is a degenerative neurological disease that affects the pyramidal pathway along its first and second motor neurons and results in the progressive loss of bulbar and limb function. Therefore, the existence of lateral sclerosis involves the damage of projection axons of the first motor neuron and amyotrophic damage of the second motor neuron. The diagnosis of this pathology is primarily clinical [46], classically reflected in the criteria of El Escorial of 199847]. Moreover, in 2008, electromyographic criteria were defined as a diagnostic tool for second motor neuron injury, despite the absence of semiological findings pathologically (Awaji criteria) [48]. Most ALS cases are sporadic; however, around $10 \%$ of cases may be familial due to mutations in genes, including those for $\mathrm{Cu} / \mathrm{Zn}$ superoxide dismutase 1 (SOD1), dynactin, TAR DNA binding protein 43 (TDP-43), and chromosome 9 open reading frame 72 (C9orf72) [49]. Although the latest research suggests that p38 and JNK MAPK play a determinant role in ALS ${ }^{[50]}$, ERK pathway alteration is also related, since SOD1(G93A) transgenic mice present a dysregulation in axonal transport associated with the down-regulation of ERK correlating with the up-regulation of JNK and caspase-8 $[\underline{24]}$.

Huntington's disease (HD) is one of nine autosomal dominant neurological diseases caused by an expansion mutation of CAG triplets encoding polyglutamine (polyQ) sequences in N-terminal domains. It affects 3-7 cases per 100,000 of the Western Europe population, and its symptoms include motor disorders (chorea and stiffness, among others), cognitive disorders (subcortical dementia), and psychological disorders (such as irritability and depression), which end with the death of patients. While the wild-type huntingtin $(\mathrm{Htt})$ protein modulates intracellular vesicular trafficking and neuronal development, mutant $\mathrm{Htt}$, with an elongated polyQ domain, generates toxic $\mathrm{N}$-terminal fragments after undergoing proteolytic processing [51].

Mutant Htt presents kinase downstream ERK deficiency involved in transcriptional dysregulation and by triggering striatal degeneration, it also decreases the response to cortico-striatal BDNF signaling and downregulates ERKdependent glutamate transporter expression, increasing cells susceptible to glutamatergic excitotoxicity [25][26]. 


\section{Prion Diseases}

A prion is the altered form of a $23-\mathrm{kDa}$ constitutive protein ( $\operatorname{PrP}$ in mammals) that has lost its normal function, but has acquired the property of transforming the standard form into a pathological form. This protein has a regular conformation called PrPc, encoded by a gene (PRNP) localized to human chromosome 20. In prion pathologies or prionopathies, an altered isoform originating as a result of the incomplete proteolysis of PrPc, called PrPsc, tends to form amyloid aggregates in the form of plaques in the brain. Prionopathies are disorders of the conformation of proteins, which manifest themselves as spongiform encephalopathy in animals, such as scrapie, and as neurodegenerative diseases in humans. The accumulation of PrPsc causes the involvement of the gray matter with neuronal death, gliosis, and spongiform changes. Activated microglia is a classic hallmark of neuroinflammation associated with prions, as these cells phagocytize and eliminate amyloid plaques [52][53]. As a part of the neuroinflammatory scenario, activated microglial cells regulate MAPK signaling pathways ${ }^{[54]}$.

Scrapie-infected hamster's brains present an up-regulation of both pJNK and pERK ${ }^{[55]}$. ERK is neuroprotective following prion infection, since the inhibition of phospho-ERK triggered the death of scrapie-infected cells. Even more, membrane-resident PrP proteins trigger phospho-ERK activation [27]. After prion infection, there is an increased level of the phospho-ERK complex, but this is also related to a decrease in MEK complex activation, suggesting a divergent action of some phosphatases on ERK1/2 upon chronic prion infection [28].

\section{References}

1. Richard M Ransohoff; How neuroinflammation contributes to neurodegeneration. Science 2016, 353, 777-783, 10.1126/science.aag2590.

2. V. Hugh Perry; James A. R. Nicoll; Clive Holmes; Microglia in neurodegenerative disease. Nature Reviews Neurology 2010, 6, 193201, 10.1038/nrneurol.2010.17.

3. MacKenzie A. Michell-Robinson; Hanane Touil; Luke Healy; David R. Owen; Bryce A. Durafourt; Amit Bar-Or; Jack P. Antel; Craig Moore; Roles of microglia in brain development, tissue maintenance and repair. Brain 2015, 138, 1138-1159, 10.1093/brain/awv066.

4. Lysia S. Forno; The Neuropathology Of Parkinson'S Disease. Progress in Parkinson Research 1988, 1, 11-21, 10.1007/978-1-46130759-4_2.

5. Amin Karimi-Moghadam; Saeid Charsouei; Benjamin Bell; Mohammad Reza Jabalameli; Parkinson Disease from Mendelian Forms to Genetic Susceptibility: New Molecular Insights into the Neurodegeneration Process.. Cellular and Molecular Neurobiology 2018, 38, 1153-1178, 10.1007/s10571-018-0587-4.

6. Dena G. Hernandez; Xylena Reed; Andrew B. Singleton; Genetics in Parkinson disease: Mendelian versus non-Mendelian inheritance.. Journal of Neurochemistry 2016, 139, 59-74, 10.1111/jnc.13593.

7. Manish Verma; Erin K. Steer; Charleen T. Chu; ERKed by LRRK2: a cell biological perspective on hereditary and sporadic Parkinson's disease.. Biochimica et Biophysica Acta (BBA) - Molecular Cell Research 2013, 1842, 1273-81, 10.1016/j.bbadis.2013.11.005.

8. Peter Reinhardt; Benjamin Schmid; Lena F. Burbulla; David C. Schöndorf; Lydia Wagner; Michael Glatza; Susanne Höing; Gunnar Hargus; Susanna A. Heck; Ashutosh Dhingra; et al.Guangming WuStephan MüllerKathrin BrockmannTorsten KlubaMartina MaiselRejko KrügerDaniela BergYaroslav TsytsyuraCora S. ThielOlympia-Ekaterini PsathakiJürgen KlingaufTanja KuhlmannMarlene KlewinHeiko MullerThomas GasserHans R. SchölerJared Sterneckert Genetic Correction of a LRRK2 Mutation in Human iPSCs Links Parkinsonian Neurodegeneration to ERK-Dependent Changes in Gene Expression. Cell Stem Cell 2013, 12, 354-367, 10.1016/j.stem.2013.01.008.

9. Linda R. White; Mathias Toft; Sylvia N. Kvam; Matthew J. Farrer; Jan O. Aasly; MAPK-pathway activity, Lrrk2 G2019S, and Parkinson's disease. Journal of Neuroscience Research 2007, 85, 1288-1294, 10.1002/jnr.21240.

10. Scott M. Kulich; Charleen T. Chu; Sustained extracellular signal-regulated kinase activation by 6-hydroxydopamine: implications for Parkinson's disease. Journal of Neurochemistry 2001, 77, 1058-1066, 10.1046/j.1471-4159.2001.00304.x.

11. Mohamed Rafiuddin Ahmed; Mithya Jayakumar; Mohamed Sohail Ahmed; Alsu I. Zamaleeva; Juan Tao; Eric Howard Li; Judith K. Job; Christopher Pittenger; Hiroshi Ohtsu; Jayakumar Rajadas; et al. Pharmacological antagonism of histamine H2R ameliorated LDOPA-induced dyskinesia via normalization of GRK3 and by suppressing FosB and ERK in PD.. Neurobiology of Aging 2019, 81, 177-189, 10.1016/j.neurobiolaging.2019.06.004.

12. J H Zhu; A M Gusdon; Huseyin Cimen; B Van Houten; E Koç; Charleen T. Chu; Impaired mitochondrial biogenesis contributes to depletion of functional mitochondria in chronic MPP+ toxicity: dual roles for ERK1/2. Cell Death \& Disease 2012, 3, e312-e312, 10.1038/cddis.2012.46.

13. Yi Li; Ning Chen; Chao Wu; Yongquan Lu; Ge Gao; Chunli Duan; Hui Yang; Lingling Lu; Galectin-1 attenuates neurodegeneration in 
Parkinson's disease model by modulating microglial MAPK/IKB/NFKB axis through its carbohydrate-recognition domain.. Brain, Behavior, and Immunity 2019, 83, 214-225, 10.1016/j.bbi.2019.10.015.

14. Jian-Hui Zhu; Scott M. Kulich; Tim D. Oury; Charleen T. Chu; Cytoplasmic Aggregates of Phosphorylated Extracellular SignalRegulated Protein Kinases in Lewy Body Diseases. The American Journal of Pathology 2002, 161, 2087-2098, 10.1016/s00029440(10)64487-2.

15. K T Dineley; M Westerman; D Bui; K Bell; K H Ashe; J D Sweatt; Beta-amyloid activates the mitogen-activated protein kinase cascade via hippocampal alpha7 nicotinic acetylcholine receptors: In vitro and in vivo mechanisms related to Alzheimer's disease.. The Journal of Neuroscience 2001, 21, 4125-33.

16. Karen A. Bell; Kenneth J O'riordan; J. David Sweatt; Kelly T. Dineley; MAPK recruitment by beta-amyloid in organotypic hippocampal slice cultures depends on physical state and exposure time. Journal of Neurochemistry 2004, 91, 349-361, 10.1111/j.14714159.2004.02722.x.

17. Claudio Russo; Virginia Dolcini; Serena Salis; Valentina Venezia; Elisabetta Violani; Pia Carlo; Nicola Zambrano; Tommaso Russo; Gennaro Schettini; Signal Transduction through Tyrosine-Phosphorylated Carboxy-Terminal Fragments of APP via an Enhanced Interaction with Shc/Grb2 Adaptor Proteins in Reactive Astrocytes of Alzheimer's Disease Brain. Annals of the New York Academy of Sciences 2002, 973, 323-333, 10.1111/j.1749-6632.2002.tb04660.x.

18. W.N. Yang; K.G. Ma; Xiaolong Chen; L.L. Shi; G. Bu; X.D. Hu; H. Han; Y. Liu; Y.H. Qian; Mitogen-activated protein kinase signaling pathways are involved in regulating $\alpha 7$ nicotinic acetylcholine receptor-mediated amyloid- $\beta$ uptake in SH-SY5Y cells. Neuroscience 2014, 278, 276-290, 10.1016/j.neuroscience.2014.08.013.

19. N Hettiarachchi; Mark L. Dallas; M Al-Owais; Heledd H. Jarosz-Griffiths; Nigel M. Hooper; J Scragg; J Boyle; Chris Peers; Heme oxygenase-1 protects against Alzheimer's amyloid- $\beta(1-42)$-induced toxicity via carbon monoxide production.. Cell Death \& Disease 2014, 5, e1569-e1569, 10.1038/cddis.2014.529.

20. Charles E. Evans; Rhian S. Thomas; Thomas J. Freeman; Martha Hvoslef-Eide; Mark A. Good; Emma J. Kidd; Selective reduction of APP-BACE1 activity improves memory via NMDA-NR2B receptor-mediated mechanisms in aged PDAPP mice.. Neurobiology of Aging 2018, 75, 136-149, 10.1016/j.neurobiolaging.2018.11.011.

21. Grigory Krapivinsky; Luba Krapivinsky; Yunona Manasian; Anton Ivanov; Roman Tyzio; Christophe Pellegrino; Yehezkel Ben-Ari; David E. Clapham; Igor Medina; The NMDA Receptor Is Coupled to the ERK Pathway by a Direct Interaction between NR2B and RasGRF1. Neuron 2003, 40, 775-784, 10.1016/s0896-6273(03)00645-7.

22. Antonella Caccamo; Smita Majumder; Arlan Richardson; Randy Strong; Salvatore Oddo; Molecular Interplay between Mammalian Target of Rapamycin (mTOR), Amyloid- $\beta$, and Tau. Journal of Biological Chemistry 2010, 285, 13107-13120, 10.1074/jbc.M110.100420.

23. Charles E. Evans; J. Scott Miners; Giulia Piva; Christine L. Willis; David M. Heard; Emma J. Kidd; Mark A. Good; Patrick G Kehoe; ACE2 activation protects against cognitive decline and reduces amyloid pathology in the Tg2576 mouse model of Alzheimer's disease. Acta Neuropathologica 2020, 139, 485-502, 10.1007/s00401-019-02098-6.

24. Eran Perlson; Goo-Bo Jeong; Jennifer L. Ross; Ram Dixit; Karen E. Wallace; Robert G. Kalb; Erika L.F. Holzbaur; A switch in retrograde signaling from survival to stress in rapid-onset neurodegeneration.. The Journal of Neuroscience 2009, 29, 9903-17, 10.1523/JNEUROSCI.0813-09.2009.

25. László Bodai; J. Lawrence Marsh; A novel target for Huntington's disease: ERK at the crossroads of signaling. The ERK signaling pathway is implicated in Huntington's disease and its upregulation ameliorates pathology.. BioEssays 2012, 34, 142-8.

26. Emmanuel Roze; Sandrine Bettuing; Carole Deyts; Estelle Marcon; Karen Brami-Cherrier; Christiane Pagès; Sandrine Humbert; Karine Merienne; Jocelyne Caboche; Mitogen- and stress-activated protein kinase-1 deficiency is involved in expanded-huntingtininduced transcriptional dysregulation and striatal death. The FASEB Journal 2007, 22, 1083-1093, 10.1096/fj.07-9814.

27. Kay M. Uppington; David Brown; Resistance of cell lines to prion toxicity aided by phospho-ERK expression. Journal of Neurochemistry 2008, 105, 842-852, 10.1111/j.1471-4159.2007.05192.x.

28. Rachel A. Lacasse; James F. Striebel; Cynthia Favara; Lisa Kercher; Bruce Chesebro; Role of Erk1/2 activation in prion disease pathogenesis: Absence of CCR1 leads to increased Erk1/2 activation and accelerated disease progression. Journal of Neuroimmunology 2008, 196, 16-26, 10.1016/j.jneuroim.2008.02.009.

29. Roberto Di Maio; Eric K. Hoffman; Emily M. Rocha; Matthew T. Keeney; Laurie H. Sanders; Briana R. De Miranda; Alevtina Zharikov; Amber Van Laar; Antonia F. Stepan; Thomas A. Lanz; et al.Julia K. KoflerEdward A BurtonDario R. AlessiTeresa G. HastingsJ. Timothy Greenamyre LRRK2 activation in idiopathic Parkinson's disease. Science Translational Medicine 2018, 10, eaar5429, 10.1126/scitransImed.aar5429.

30. Edward D. Plowey; Salvatore J. Cherra; Yong-Jian Liu; Charleen T. Chu; Role of autophagy in G2019S-LRRK2-associated neurite shortening in differentiated SH-SY5Y cells.. Journal of Neurochemistry 2008, 105, 1048-56, 10.1111/j.1471-4159.2008.05217.x.

31. Cristina Gómez-Santos; Isidre Ferrer; Julia Reiriz; Francesc Viñals; Marta Barrachina; Santiago Ambrosio; MPP+ increases $\alpha$ synuclein expression and ERK/MAP-kinase phosphorylation in human neuroblastoma SH-SY5Y cells. Brain Research 2002, 935, 32-39, 10.1016/s0006-8993(02)02422-8.

32. Sumit Sarkar; Edward Lu; James Raymick; Joseph Hanig; Qiang Gu; ERK/MAP Kinase Activation is Evident in Activated Microglia of 
the Striatum and Substantia Nigra in an Acute and Chronically-Induced Mouse Model of Parkinson's Disease. Current Neurovascular Research 2019, 15, 336-344, 10.2174/1567202616666181123152601.

33. Sagar H. Barage; Kailas D. Sonawane; Amyloid cascade hypothesis: Pathogenesis and therapeutic strategies in Alzheimer's disease. Neuropeptides 2015, 52, 1-18, 10.1016/j.npep.2015.06.008.

34. George S. Bloom; Amyloid-beta and tau: the trigger and bullet in Alzheimer disease pathogenesis. JAMA Neurology 2014, 71, 505508, 10.1001/jamaneurol.2013.5847.

35. J. Pozueta; Roger Lefort; Michael L Shelanski; Synaptic changes in Alzheimer's disease and its models. Neuroscience 2013, 251, 51-65, 10.1016/j.neuroscience.2012.05.050.

36. E Masliah; E Rockenstein; Genetically altered transgenic models of Alzheimer's disease.. Journal of neural transmission. Supplementum 2000, 59, 175-183.

37. M W Decker; The effects of aging on hippocampal and cortical projections of the forebrain cholinergic system.. Brain Research 1987, 434, 423-438.

38. Elodie Bruel-Jungerman; Paul J. Lucassen; Fiona Francis; Cholinergic influences on cortical development and adult neurogenesis. Behavioural Brain Research 2011, 221, 379-388, 10.1016/j.bbr.2011.01.021.

39. Heather D. VanGuilder; Han Yan; Julie A. Farley; William E. Sonntag; Willard M Freeman; Aging alters the expression of neurotransmission-regulating proteins in the hippocampal synaptoproteome.. Journal of Neurochemistry 2010, 113, 1577-88, 10.1111/j.1471-4159.2010.06719.x.

40. Michael T. Heneka; Monica J. Carson; Joseph El Khoury; Gary E. Landreth; Frederic Brosseron; Uglas L. Feinstein; Andreas H. Jacobs; Tony Wyss-Coray; Javier Vitorica; Richard M. Ransohoff; et al.Karl HerrupSally A. FrautschyBente FinsenGuy C. BrownAlexei VerkhratskyKoji YamanakaJari KoistinahoEicke LatzAnnett HalleGabor C. PetzoldTerrence TownDave MorganMari L. ShinoharaV. Hugh PerryClive HolmesNicolas G. BazanDavid J. BrooksStéphane HunotBertrand JosephNikolaus DeigendeschOlga GaraschukErik BoddekeCharles A. DinarelloJohn C. BreitnerGreg M. ColeUglas T. GolenbockMarkus P. KummerFrederik Brosseron Neuroinflammation in Alzheimer's disease.. The Lancet Neurology 2015, 14, 388-405, 10.1016/S1474-4422(15)70016-5.

41. Xiongwei Zhu; Rudolph J Castellani; Atsushi Takeda; Akihiko Nunomura; Craig S Atwood; George Perry; Mark A Smith; Differential activation of neuronal ERK, JNK/SAPK and p38 in Alzheimer disease: the 'two hit' hypothesis. Mechanisms of Ageing and Development 2001, 123, 39-46, 10.1016/s0047-6374(01)00342-6.

42. E. Giraldo; Ana Lloret; Tanja Fuchsberger; J. Viña; $A \beta$ and tau toxicities in Alzheimer's are linked via oxidative stress-induced $p 38$ activation: protective role of vitamin E.. Redox Biology 2014, 2, 873-7, 10.1016/j.redox.2014.03.002.

43. Magdalena Cieślik; Grzegorz Arkadiusz Czapski; Joanna B. Strosznajder; The Molecular Mechanism of Amyloid $\beta 42$ Peptide Toxicity: The Role of Sphingosine Kinase-1 and Mitochondrial Sirtuins. PLOS ONE 2015, 10, e0137193,

10.1371/journal.pone.0137193.

44. Hong-Quan Wang; Yuxia Xu; Cuiqing Zhu; Upregulation of Heme Oxygenase-1 by Acteoside Through ERK and PI3 K/Akt Pathway Confer Neuroprotection Against Beta-Amyloid-Induced Neurotoxicity. Neurotoxicity Research 2011, 21, 368-378, 10.1007/s12640011-9292-5.

45. L.P. Rowland; How amyotrophic lateral sclerosis got its name: the clinical-pathologic genius of Jean-Martin Charcot.. Archives of Neurology 2001, 58, 512-5.

46. Orla Hardiman; Leonard H. Van Den Berg; Matthew C. Kiernan; Clinical diagnosis and management of amyotrophic lateral sclerosis. Nature Reviews Neurology 2011, 7, 639-649, 10.1038/nrneurol.2011.153.

47. Benjamin Rix Brooks; El escorial World Federation of Neurology criteria for the diagnosis of amyotrophic lateral sclerosis. Journal of the Neurological Sciences 1994, 124, 96-107, 10.1016/0022-510x(94)90191-0.

48. Mamede De Carvalho; Michael Swash; Awaji diagnostic algorithm increases sensitivity of El Escorial criteria for ALS diagnosis. Amyotrophic Lateral Sclerosis 2009, 10, 53-57, 10.1080/17482960802521126.

49. Kevin Boylan; Familial Amyotrophic Lateral Sclerosis.. Neurologic Clinics 2015, 33, 807-30, 10.1016/j.ncl.2015.07.001.

50. Katherine L. Gibbs; Bernadett Kalmár; Elena R. Rhymes; Alexander D. Fellows; Mahmood Ahmed; Paul Whiting; Ceri H. Davies; Linda Greensmith; Giampietro Schiavo; Inhibiting p38 MAPK alpha rescues axonal retrograde transport defects in a mouse model of ALS.. Cell Death \& Disease 2018, 9, 596, 10.1038/s41419-018-0624-8.

51. Akira Sawa; Eiichiro Nagata; Siobhan Sutcliffe; Pratima Dulloor; Matthew B. Cascio; Yuji Ozeki; Sophie Roy; Christopher A. Ross; Solomon H. Snyder; Huntingtin is cleaved by caspases in the cytoplasm and translocated to the nucleus via perinuclear sites in Huntington's disease patient lymphoblasts. Neurobiology of Disease 2005, 20, 267-274, 10.1016/j.nbd.2005.02.013.

52. Adriano Aguzzi; Asvin Lakkaraju; Press Enter Key For Correspondence Information; Cell Biology of Prions and Prionoids: A Status Report. Trends in Cell Biology 2016, 26, 40-51, 10.1016/j.tcb.2015.08.007.

53. Adriano Aguzzi; Caihong Zhu; Microglia in prion diseases.. Journal of Clinical Investigation 2017, 127, 3230-3239, 10.1172/JCI90605.

54. Junghyung Park; Ju-Sik Min; Bokyung Kim; Un-Bin Chae; Jong Won Yun; Myung-Suk Choi; Il-Keun Kong; And Kyu-Tae Chang; Hyo Jin Lee; Mitochondrial ROS govern the LPS-induced pro-inflammatory response in microglia cells by regulating MAPK and NF-KB pathways. Neuroscience Letters 2015, 584, 191-196, 10.1016/j.neulet.2014.10.016. 
55. Hyun-Pil Lee; Yong-Cheol Jun; Jin-Kyu Choi; Jae-II Kim; Richard I. Carp; Yong-Sun Kim; Activation of mitogen-activated protein kinases in hamster brains infected with 263K scrapie agent. Journal of Neurochemistry 2005, 95, 584-593, 10.1111/j.14714159.2005.03429.x.

\section{Keywords}

learning; memory; hippocampus; septum; synapse; Amyotrophic lateral sclerosis; Huntington's disease; Prion diseases; Alzheimer's disease; Parkinson's disease 